\title{
'The National Security Strategy of the USA' ou la rencontre improbable de Grotius, Carl Schmitt et Philip K. Dick
}

\section{Francesco Ragazzi}

\section{(2) OpenEdition \\ Journals}

Édition électronique

URL : http://journals.openedition.org/conflits/1648

DOI : $10.4000 /$ conflits. 1648

ISSN : 1777-5345

Éditeur :

CCLS - Centre d'études sur les conflits lilberté et sécurité, L'Harmattan

Édition imprimée

Date de publication : 1 décembre 2004

Pagination : 141-156

ISBN : 2-7475-7598-X

ISSN : 1157-996X

Référence électronique

Francesco Ragazzi, «'The National Security Strategy of the USA' ou la rencontre improbable de Grotius, Carl Schmitt et Philip K. Dick », Cultures \& Conflits [En ligne], 56 | hiver 2004, mis en ligne le 07 janvier 2010, consulté le 30 mars 2021. URL : http://journals.openedition.org/conflits/1648 ; DOI : https:// doi.org/10.4000/conflits. 1648

Ce document a été généré automatiquement le 30 mars 2021

Creative Commons License 


\title{
'The National Security Strategy of the USA' ou la rencontre improbable de Grotius, Carl Schmitt et Philip K. $\operatorname{Dick}^{1}$
}

\author{
Francesco Ragazzi
}

« Supprimez la justice, que sont les empires, si ce n'est de grands brigandages? $»^{2}$

1 «C'est une expérience inquiétante que d'écrire quelque chose dans un roman en croyant que c'est une pure fiction, et d'apprendre par la suite - parfois des années plus tard - que c'est vrai ». C'est ce qu'écrivait en 1978 l'auteur de science-fiction Philip K. Dick, dans « How to build a Universe that doesn't fall apart two days later ${ }^{3}$, un texte illuminé sur les rapports possibles entre « fiction » et « réalité ». Or il nous a semblé frappant de constater à quel point les textes de Philip K. Dick ont su anticiper un grand nombre de problématiques futures, une capacité probablement liée à la nature même du genre de la sciencefiction : plus qu'imaginer des technologies du futur (décrire des soucoupes volantes ou des martiens envahisseurs), l'œuvre de Dick a consisté avant tout à imaginer et mettre en scène, à partir de données du présent, la possibilité de différents mondes sociaux. La croyance aveugle de l'Homme en une science humaine prédictive, les enjeux de pouvoir liés à la connaissance, l'idée que la « réalité » puisse être un construit - et un construit manipulable - sont autant de problèmes que Philip K. Dick partage sans le savoir avec les débats et les réflexions épistémologiques en Relations Internationales, et en Sciences Sociales plus généralement. Parmi les textes de Dick, une nouvelle particulièrement parlante aujourd'hui - et ce n'est pas seulement parce qu'un film en a été tiré - est sans doute « Rapport Minoritaire " (Minority Report). Elle est avant tout intéressante par sa capacité étonnante à parler d'un autre document d'actualité : la Stratégie de Sécurité Nationale des Etats-Unis (The National Security Strategy of The U.S.A. ${ }^{4}$ ), publiée en septembre 2002. C'est à travers la nouvelle de Dick, et de quelques autres textes, que nous essaierons de lire ce document de la stratégie des Etats-Unis. 
2 Il ne s'agira pas ici de dire s'il faut croire ou non au discours américain en matière de sécurité, ou si en réalité l'action des Etats-Unis serait guidée par des intérêts différents de leur discours, mais au contraire de comprendre l'argumentaire de la doctrine américaine en matière de sécurité. On s'arrêtera plus précisément sur l'articulation de trois idées : l'idée de la l'attaque 'préemptive' d'une part, l'idée explicitement évoquée de la guerre juste d'autre part, et enfin l'idée de l'état d'exception à l'échelle internationale. Sans grande surprise, la façon dont ces idées s'articulent nous permet de saisir la dimension non seulement unilatérale mais bien impériale de la logique de sécurité des Etats-Unis.

\section{La guerre 'préemptive' : quand « l'acte criminel proprement dit relève de la métaphysique $\star^{5}$}

3 Anderton, préfet de police de l'Alliance Fédérale du Bloc Occidental, a mis au point un système qui permet de prévoir le futur - quelques semaines seulement - à l'aide des pouvoirs surnaturels de trois êtres anormaux, les "précogs». Leurs visions sont analysées et comparées, et depuis trente ans, le département de Précrime se charge d'identifier et d'arrêter les meurtriers avant qu'ils ne passent à l'acte. Le dernier crime date d'il y a cinq ans, et la société de l'Alliance s'est habituée à vivre dans un monde sans meurtres. La seule contrepartie à l'abolition du système punitif postcrime (jugé peu dissuasif, et peu réconfortant pour les victimes) tient dans l'établissement de camps où sont enfermés des milliers de criminels potentiels. Et c'est bien là un des premiers paradoxes du système : "L'inconvénient fondamental, du point de vue juridique, inhérent à la méthodologie de Précrime ne vous a probablement pas échappé non plus [explique Anderton à l'un de ses collègues]. Nous arrêtons des individus qui n'ont nullement enfreint la loi. (...) Donc l'acte criminel proprement dit ne relève strictement que de la métaphysique. C'est nous qui proclamons ces gens coupables. Eux se prétendent éternellement innocents. Et en un sens, ils sont innocents $»^{6}$. Le système est donc un système prophylactique : pour éviter le risque de meurtre, on enferme les potentiels criminels.

Cependant, on comprend au cours de la nouvelle - peu importe ici l'histoire en ellemême - que la machine n'est pas complètement infaillible; elle laisse une certaine place à l'incertitude. En effet, si les trois semi-humains en hibernation peuvent prédire l'avenir, leurs prévisions ne sont pas toujours unanimes. Il faut donc que deux des prévisions convergent pour que, statistiquement, les cartes perforées permettent au système de prévoir avec un certain degré de certitude l'avènement d'un meurtre ${ }^{7}$. Il y a donc toujours l'un des trois rapports qui est écarté, le rapport minoritaire. En effet, toujours d'après la nouvelle, le système fonctionne d'après la théorie des «futurs multiples », c'est-à-dire l'existence de plusieurs sillons temporels : il n'y a pas un seul futur possible (sinon on ne pourrait agir pour prévenir les crimes) mais bien une série de possibilités. Tout devient possible donc pour celui qui à la fois connaît le futur et peut agir sur lui.

5 En somme, les précogs permettent d'obtenir une photographie des possibles à un moment précis du présent. Ils peuvent donc être en discordance du fait qu'ils expriment des possibilités, mais également du fait qu'ils incluent leurs propres prévisions comme des actes ayant des conséquences dans le futur (il y a donc une dimension réflexive à ces prévisions). Les précogs prévoient donc à la fois le meurtre et le non-meurtre, le crime et le non-crime, c'est-à-dire qu'ils mettent à jour des scénarios 
possibles qui deviennent des probables. C'est donc plus la croyance humaine dans l'infaillibilité du système que le système lui-même qui représente le réel danger, et c'est en effet cela le centre de l'intrigue. Incertitude, réduction de l'incertitude, prévention du risque, action préventive, voire préemptive... ni le registre des préoccupations ni le vocabulaire même de la nouvelle ne sont très éloignés de notre propos.

6 «Nous ne pouvons laisser nos ennemis frapper les premiers" ${ }^{8}$. Dans le National Security Strategy de la Maison Blanche de septembre 2002, une page (peut-être temporaire) est tournée dans la conception que se font les Etats-Unis de leur sécurité. L'attaque par surprise, cette variable incertaine que toute l'histoire de la dissuasion a essayé d'éviter ${ }^{9}$, devient la règle. Désormais, il ne s'agit plus d'attendre que la menace se concrétise, mais il faut pouvoir maîtriser le temps du conflit. La sécurité américaine devient une sécurité préemptive : «Etant donnés les buts des Etats-voyous [rogue states] et des terroristes, les Etats-Unis ne peuvent plus compter uniquement sur une attitude réactive comme nous l'avons fait par le passé. L'incapacité à dissuader un attaquant potentiel, l'immédiateté des menaces d'aujourd'hui, l'ampleur du mal potentiel qui peut être causé par le choix des armes de nos adversaires ne nous permettent pas defaire ce choix $»^{10}$.

7 Comment se justifie ce changement ? Dans Minority Report, le changement du système postcrime à précrime était justifié dans la nouvelle de Dick par deux présupposés : tout d'abord, l'idée qu'il n'y a rien à prouver ni à contester : la Précrime connaît la vérité, et qui plus est connaît le futur. Comment? A travers l'infaillibilité (prétendue) des précogs. La seule justification possible pour l'intervention, c'est le caractère infaillible de la prévision: il n'y aurait d'arrestation préventive juste si elle ne s'imposait pas comme une nécessité, s'il n'était pas inévitable que le crime se produise. Mais il y a une seconde idée : puisque le crime est inévitable, et qu'il s'agit d'un meurtre - donc d'un acte criminel irréversible et destructeur - il est juste que la police intervienne. L'action de la police Précrime, comme le dit Anderton est un «service public », un «bien pour la société ». La raison pour laquelle on accepte d'envoyer tous ces potentiels criminels dans les camps, c'est que l'on estime que l'action de la Précrime est juste. Doit-on laisser commettre des crimes, puisqu'il n'y a pas de doute sur le fait qu'ils seront commis? Et quand bien même il ne s'agirait que d'un risque, serait-il justifié de ne pas agir en premier pour le prévenir?

8 Cela relève donc du bon sens que de remettre en cause l'organisation du système punitif postcrime: détruire les principes juridiques du fonctionnement pénal est justifié par l'infaillibilité du nouveau système de prévision. La menace n'a pas changé de nature, mais c'est la capacité à la déjouer qui incite à une réorganisation des principes juridiques.

9 Pour la Maison Blanche, ce ne sont pas les mêmes raisons qui demandent de repenser la conception de la sécurité. Le changement est fondé sur le changement de la nature de la menace, c'est-à-dire de la désignation du nouvel ennemi : le terrorisme. Quelles en sont les caractéristiques?

10 Tout d'abord, la menace provient aujourd'hui des «Etats-voyous » (rogue states) et des terroristes. Associés, les Etats territoriaux et les organisations violentes déterritorialisées présentent la capacité de déclencher des attaques de façon inattendue, imprévisible. Etrangement, les développements sur la lutte contre le terrorisme abandonnent rapidement les organisations terroristes pour ne s'intéresser qu'à la politique à mener contre les Etats. Qu'est-ce qui fait que ces Etats ont un comportement inattendu? Ils ne sont pas démocratiques, ils «brutalisent leurs propres 
peuples »"11, ils n'ont "pas d'égards pour le droit international», ils sont "déterminés à acquérir des armes de destruction massive", ils soutiennent le terrorisme, et enfin "détestent les Etats-Unis et tout ce qu'ils représentent ${ }^{12}$. Ce qui est implicite dans cette liste de caractéristiques, c'est avant tout le caractère imprévisible du comportement d'organisations qui n'auraient pas une rationalité équivalente à celle des (autres) Etats, sous entendus comme les Etats libéraux - ou encore comme les Etats-Unis, leurs amis et leurs alliés. Ce qui rend les organisations " terroristes » invulnérables (le raisonnement ne s'inquiète pas de ce paradoxe) c'est en outre le fait de n'appartenir à aucun Etat: "Les concepts traditionnels de dissuasion n'auront pas d'effets contre un ennemi terroriste dont la tactique avouée est de détruire gratuitement et de cibler des innocents; [un ennemi] dont les soi-disant soldats cherchent le martyre dans la mort, et dont la protection la plus efficace est de ne pas être lié un Etat [statelessness] $»^{13}$.

Un deuxième aspect important de cette menace, c'est que non seulement ces Etats sont imprévisibles par nature, irrationnels, mais qu'ils sont « au carrefour entre le radicalisme [religieux] et la technologie $»^{14}$ : grâce aux nouvelles technologies, ils sont capables de mettre au point, sans que l'on aie le temps de s'en apercevoir, des armes de destruction massive. Et il devient non seulement de plus en plus difficile de percevoir la formation de la menace, mais sans besoin de grands moyens, les ennemis peuvent attaquer par surprise et par tromperie (deceit), à n'importe quel moment : «(...) ils agissent par des actes de terreur et, potentiellement, sur l'utilisation d'armes de destruction massive - des armes qui peuvent facilement être cachées, livrées secrètement et utilisées sans avertissement ${ }^{15}$. Enfin, dernière caractéristique, ces attaques peuvent être extrêmement meurtrières, comme en témoignent les événements du onze septembre. Mais les nouveaux ennemis peuvent aussi faire usage d'armes de destruction massive, ce qui rendrait l'attaque encore plus effroyable.

12 Le « cocktail » qui permet d'expliquer ce nouveau tournant dans la réponse à apporter à ces menaces a été décrit par Pierre Hassner ${ }^{16}$ comme une combinaison de trois éléments. Premièrement, un nouveau sentiment de vulnérabilité, lié aux attentats du onze septembre: "Ce que nous chérissons le plus, - notre liberté, nos villes, nos systèmes de mouvement et de vie moderne - sont vulnérables au terrorisme $»^{17}$. Deuxièmement, il s'agirait d'une incompréhension devant l'hostilité gratuite du terrorisme et le manque de solidarité internationale devant l'ampleur du phénomène qui les a accablés. Enfin, il faudrait considérer le sentiment d'une puissance inégalée, infaillible : «Les Etats-Unis possèdent une force et une influence inégalées et sans précédent dans le monde » ${ }^{18}$. La " Guerre contre le terrorisme » et son caractère préemptif deviennent subitement une politique étrangère claire et simple, digne de la "clarté morale » (moral clarity) affichée par le président américain. Simple et universelle comme la guerre contre le communisme d'antan, elle est présentée comme une position de bon sens ${ }^{19}$; en emportant l'adhésion d'une majorité d'américains, elle correspond facilement enfin aux croyances du « New Born Christian » qu'est George Bush. Le «bien contre le mal », cette vision du monde qui n'admet pas de "moralités différentes ${ }^{20}$ est celle de la désignation du terrorisme comme l'ennemi absolu. La spécificité de la désignation de l'ennemi - à la différence de celle de la désignation de l'adversaire - pour reprendre les distinctions d'Edelman ${ }^{21}$, c'est que l'humanité de l'ennemi est niée dans sa propre nature. L'image de l'autre est construite comme celle de quelqu'un qui ne pourra jamais "être comme nous ", dans la mesure où ses traits physiques, mentaux et culturels sont fondamentalement différents des notres, donc dangereux. La seule solution pour résoudre la confrontation avec un ennemi est sa destruction physique puisque sa nature même est une menace, et 
quand bien même elle n'est pas imminente, elle est potentielle. Autant donc agir tout de suite. Et cette nécessité d'agir tout de suite, de façon préemptive, redéfinit profondément la doctrine de la sécurité américaine.

\section{La révision du droit international et la justification par la guerre juste}

13 La doctrine de la préemption ne prolonge pas la dialectique traditionnelle, en matière de politique étrangère, entre le "wilsonisme » et "réalisme», mais détruit de l'intérieur les règles écrites (droit international, alliances) et/ou non écrites (anarchie, équilibre des puissances) de l'ordre international.

Pierre Hassner et Justin Vaïsse ${ }^{22}$ établissent une distinction entre les différentes doctrines de la politique étrangère américaine en les organisant schématiquement autour de deux axes croisés : d'une part l'axe qui va du multilatéralisme (intégration) à l'unilatéralisme (souveraineté) et d'autre part l'axe qui sépare les doctrines soutenant l'idée d'une "mission américaine", le wilsonisme, et à l'opposé les tenants de la Realpolitik, de l'équilibre des forces et de l'intérêt national. Traditionnellement - et pour aller vite - ce sont deux tendances idéaltypiques qui se sont principalement succédé à la Maison Blanche: d'une part les «internationalistes libéraux", convaincus de l'importance de la mission américaine dans le monde, de l'exceptionnalisme américain et de sa vocation universelle, les wilsoniens (on pourrait citer dans cette catégorie, à part Wilson, des présidents tels que Carter, ou Clinton); d'autre part, les tenants du strict équilibre des forces, de la stabilité internationale et d'une politique orientée vers les intérêts bien compris des Etats-Unis : les realpolitikers à la Nixon, Kissinger, James Baker ou Richard Haass. Or, l'originalité de la présidence de George W. Bush est d'avoir mis au pouvoir les partisans d'une doctrine sensiblement différente, expliquent Pierre Hassner et Justin Vaisse : les "néo-conservateurs » ou " hégémonistes ", partisans de l'idée d'intervention et de mission mondiale, mais aussi partisans de la souveraineté inébranlable des Etats-Unis. Croyant dans la force et la morale américaines, ils se placent du côté résolument "unilatéraliste » de l'axe tracé par les deux auteurs, en croyant à la nécessité de l'intervention américaine dans le monde pour assurer sa stabilité, mais sans recourir au jeu multilatéral. La doctrine de la préemption n'est donc un ni un wilsonisme, ni un containment, ni un équilibre des puissances. Ce n'est pas pour autant qu'elle ne porte pas avec elle une vision du monde et de son organisation.

La doctrine de la préemption et de la guerre contre le terrorisme ne se fonde sûrement pas sur la volonté de respecter le droit international, jugé trop contraignant et contre productif - voire sans raison d'être (irrelevant) - mais elle n'est pas non plus fondée sur le rejet de celui-ci au nom des intérêts. La doctrine de la préemption représente la volonté de détruire les normes internationales jusque-là admises (juridiques ou autres) pour les substituer par de nouvelles normes, censées répondre à la morale et à la force américaine dans le monde.

La notion de "guerre préventive» balaye donc tous les fondements du droit international. La prohibition de l'utilisation de la force pour résoudre les conflits internationaux est contenue dans l'article 2 de la Charte des Nations Unies. Seules deux exceptions sont prévues: le droit de légitime défense, dans l'article $51^{23}$, et l'action collective initiée par le Conseil de Sécurité sous le Chapitre VII. Or, aucune de ces deux 
exceptions ne couvre le droit à la guerre préventive. Comme l'explique le juriste international Dietrich Murswieck ${ }^{24}$, les Etats-Unis sont conscients que leur doctrine est en contradiction avec les principes mêmes du droit international. Mais leur intention est, si cela est possible, de modifier les principes du droit de telle sorte que l'exception (la guerre préventive) devienne la règle, en adaptant, en modifiant la notion de menace imminente : "Depuis des siècles, les lois internationales reconnaissent qu'un pays peut prendre des mesures défensives contre des forces armées qui présentent pour lui un danger immédiat, avant même qu'il n'ait subi la moindre attaque. Les législateurs, en particulier les juristes internationaux, fondent en général la légitimité de la riposte sur l'existence d'une menace imminente, le plus souvent la mobilisation visible de l'armée, de la marine, ou des forces aériennes se préparant à l'attaque. (...) Nous devons adapter le concept de menace imminente aux capacités et aux objectifs de nos adversaires d'aujourd'hui $\aleph^{25}$. Il s'agit donc du même procédé opéré dans Minority Report : suspendre un système juridique pour en proposer un nouveau, et cela pour des raisons à la fois de bon sens et de nécessité. Mais alors, qu'est-ce qui justifie l'action américaine?

17 La seule façon de justifier sa vision de la sécurité, après avoir détruit le droit international dans ses principes mêmes, pourrait être la poursuite des intérêts des Etats-Unis. Or, nous l'avons vu, ce n'est pas le propos du National Security Strategy. La vision qui y est portée refuse l'idée d'intérêts puisqu'elle propose une morale universelle. La seule justification qui peut être soutenue est alors celle de la «guerre juste». L'appel à la guerre juste, seul argument qui peut faire tenir ensemble l'argumentation du programme de la Maison Blanche, est explicite : «Nous construisons un monde de justice, ou nous vivrons dans un monde de coercition ${ }^{26}$ ou encore «Les raisons de nos actions seront claires, la force mesurée, et la cause juste $"{ }^{27}$.

Or ce que tente de faire le National Security Strategy, c'est précisément, par un tour de passe-passe, d'étendre la notion de guerre préemptive à tel point qu'elle se confonde avec celle de guerre préventive ${ }^{28}$. Or la différence - radicale - entre les deux termes est sans doute aussi vieille que le droit international. Il ne s'agit pas simplement "d'adapter", ou "d'actualiser" le terme, le but de l'argumentaire de la Maison Blanche est bien de faire passer un acte reconnu comme illégal depuis toujours pour ce qui serait une des caractéristiques de la "guerre juste». Or on peut voir, par exemple à travers Grotius, que l'idée de la guerre juste renvoie, dès les fondements du droit international, à des caractéristiques très précises, qui excluent complètement la possibilité « d'adapter » la notion de prévention. Si la notion de " guerre juste » ne naît pas avec Grotius, (il cite à ce propos l'historien romain Dion $^{29}$ ) elle trouve cependant dans son œuvre majeure, Du Droit de la Guerre et de la Paix, une formalisation importante pour le futur du Droit International.

Grotius commence par établir une distinction entre les guerres: celles qui sont motivées par l'intérêt, et celles qui sont motivées par un «principe de justice» (I.1). Parmi les dernières, «la plupart des auteurs (Baldus, Ad. Leg. 2, Cod. De Servit. et aquâ, $\mathrm{n}^{\circ} 71$, Wilh Matth., De Bello justo et licito) assignent aux guerres trois causes légitimes : la défense, le recouvrement de ce qui nous appartient et la punition ${ }^{30}$. Le chapitre I traite de la première de ces causes : «Des causes de la guerre et, premièrement, de la défense de soi-même et des biens".

20 Avant de rentrer dans une longue casuistique des différentes formes licites ou illicites de défense, Grotius s'intéresse à «La première cause d'une guerre juste [qui] est donc une injure qui n'est pas encore faite, mais qui menace le corps, ou les biens» (II.3). On retrouve 
donc ici l'idée de légitime défense liée à l'idée d'une « injure qui menace les biens », qui se retrouvera dans l'article 51 de la Charte des Nations Unies. Mais quelle est la nature de cette injure? Elle est discutée dans le paragraphe $\mathrm{V}:$ "On exige ici un péril présent, et comme compris dans un point $»^{31}$ mais "ceux-là se trompent fort, et trompent les autres, qui admettent toute crainte, quelle qu'elle soit, au droit de prévenir ainsi un assassinat ». Grotius cite également Aulu-Gelle: "Quand un gladiateur est sous les armes, la condition de la lutte est celle-ci : il tuera son adversaire, s'il le prévient; il succombera, s'il se laisse prévenir. Mais l'existence des hommes n'est pas d'ordinaire menacée par une nécessité tellement fatale et tellement imminente qu'il faille frapper le premier coup, si l'on ne veut être frappé soi-même " ${ }^{32}$. La guerre déclenchée par une crainte est donc illégitime, de même que la guerre déclenchée par la "volonté de diminuer la puissance»: "Mais que la possibilité d'être attaqués nous donne le droit d'être agresseurs, c'est contraire à tout principe d'équité. L'existence humaine est telle que jamais nous ne sommes assurés d'une complète sécurité » $(\mathrm{XVII})^{33}$.

On voit bien que Grotius avait déjà envisagé les différentes éventualités qui font l'objet de notre propos: dans la perspective grotienne, la légitime défense est contemplée parmi les causes de la guerre juste. Mais la légitime défense est définie dans un sens strict, et il s'attarde bien plus sur les dérives possibles des guerres déclenchées pour répondre à une menace qui ne serait pas, selon ses termes, « un péril présent ».

Cet aspect à double tranchant de la légitime défense, de l'attaque préventive, avait déjà trouvé une certaine résonance dans les préoccupations de la fin des années 1990 sur la " guerre humanitaire » et le " droit d'ingérence ». Dans un entretien donné à la revue Politique Internationale, Pierre Hassner avait déjà soulevé cette question, en rappelant que le principe de souveraineté nationale, qui est remis en question par le droit à l'ingérence, pouvait tout de même constituer «un frein utile aux interventions impériales $\|^{34}$. Cependant, il y a certaines situations où les Etats doivent prendre leurs responsabilités et justifier d'une « conduite d'exception ». Difficile donc pour les néoconservateurs de faire appel à cette guerre juste là, mais difficile aussi de faire appel à la «guerre juste» du droit international telle qu'elle est décrite par Grotius, puis Pufendorf et "les législateurs, en particulier les juristes internationaux » ${ }^{35}$. En ce sens - et ce n'est sans doute pas une surprise - on peut dire que la référence au droit international, ne peut que démontrer l'illégalité (du point de vue des fondements du droit international) de la guerre «préemptive ». Nous avons d'ailleurs déjà vu que c'est cette même tradition de la guerre juste (celle du droit international) qu'ils essaient de remettre en cause avec l'intention d'en modifier le contenu. Mais alors comment échapper à la parole de Saint-Augustin, citée - toujours dans Grotius - «Supprimez la justice, que sont les empires, si ce n'est de grands brigandages? » (De Civ. Dei, lib IV, cap IV) ${ }^{36}$.

\section{L'abolition de l'intérieur et de l'extérieur : l'état d'exception international ou la logique impériale}

Créer un ordre juridique qui règle un ordre non-juridique, suspendre le droit non pas pour créer «l'anarchie» mais bien pour la combattre: voilà la tâche du Préfet Anderton et de sa brigade Précrime. Mais voilà également la façon de penser l'état d'exception - au sens de Carl Schmitt - que semblent adopter les Etats-Unis, à la fois pour l'ordre interne que pour leur vision de l'ordre international : «L'état d'exception se distingue toujours de l'anarchie et du chaos et, dans un sens juridique, on y trouve encore un 
ordre, quand bien même il ne s'agit pas d'un ordre juridique $»^{37}$. Giorgio Agamben, en reprenant l'idée de l'état d'exception, insiste dans son article sur une formule clé pour comprendre l'articulation entre l'ordre juridique «normal» et l'ordre juridique de "l'état d'exception». Cette formule clé, titre d'une conférence de Jacques Derrida à New York en 1990, c'est le syntagme «force de loi ». Ce concept, qui opère une séparation entre l'efficacité de la loi et son essence formelle, permet d'envisager l'état d'exception comme "un régime de la loi dans lequel la norme vaut mais ne s'applique pas [parce qu'elle n'a pas de force] et des actes qui n'ont pas valeur de loi en acquièrent la force ».

Il faut ici préciser le raisonnement. Les réflexions d'Agamben sur l'œuvre de Schmitt concernent bien évidement l'ordre juridique interne: les concepts autour desquels s'articulent les différents raisonnements sont ceux de souverain, souveraineté, Constitution, lois, décrets. Mais bien que l'Etat reste l'élément sans lequel il nous est impossible de penser l'interne par rapport à l'externe, il faut accepter que le caractère arbitraire de ces lignes de démarcation interne/externe est de plus en plus visible ${ }^{38}$. Le dernier chapitre du National Security Strategy, «Transformer les Institutions Américaines en Matière de Sécurité pour faire face aux défis et aux opportunités du XXI ${ }^{e ̀ m e}$ siècle $»^{39}$ témoigne de la prise de conscience de cette réalité: «Aujourd'hui la distinction entre les affaires intérieures et les affaires internationales diminue ", et l'administration américaine en tire les conséquences pour la réorganisation de ses agences gouvernementales de sécuritée ${ }^{40}$. Les études sur l'état d'exception suite aux attentats du onze septembre se sont surtout concentrées sur la question de la désignation de l'ennemi intérieur, du non respect des règles de droit pour le traitement des prisonniers de la guerre en Afghanistan ou encore sur les nouvelles mesures d'exception en matière d'immigration. Mais devant le brouillage croissant de la séparation entre l'intérieur et l'extérieur, et face à l'attitude particulière du courant néo-conservateur de la Maison Blanche en matière de politique étrangère, peut-on dire que le National Security Strategy contient les bases d'un état d'exception à l'échelle internationale?

Faisons quelques pas en arrière dans le raisonnement. Dans la logique schmittienne, la séparation entre l'interne et l'externe s'opère par l'instance qu'est l'Etat (en se distinguant de la substance, le politique). A l'extérieur, «l'Etat, unité politique et centre de décision, détient et concentre un pouvoir énorme : il a la possibilité de faire la guerre et donc de disposer ouvertement de la vie d'êtres humains ${ }^{41}$; à l'intérieur, il a la tâche d'homogénéiser la société nationale et de créer un mode de fonctionnement réglé par le droit, normé. Norme à l'intérieur, exception (droit de vie et de mort sur ces propres citoyens et sur l'ennemi) à l'extérieur. Comme nous l'avons vu, l'état d'exception est la capacité, dans certaines circonstances, à désigner un ennemi intérieur, l'ennemi public. C'est-à-dire à importer la logique de guerre à l'intérieur, c'est-à-dire à suspendre le droit par un acte juridique. Or dans une logique d'interdépendance croissante, de confusion des rôles entre armée et police ${ }^{42}$, de la naissance d'un droit international efficace (donc la tentative d'une normalisation des rapports internationaux), il semble que les positions affirmées dans le National Security Strategy aillent dans le sens de l'établissement d'un état d'exception international ou encore d'une logique impériale: c'est-à-dire qu'il s'agit, pour les Etats-Unis, de s'arroger le droit de suspendre les règles de droit international pour lutter contre un ennemi intérieur (mais alors intérieur par rapport à des limites floues dessinées par l'hégémonie américaine, où le monde entier devient le contenant de ce qui est intérieur), tout en s'accordant le droit de les rétablir selon leur bon vouloir. Ce serait donc dans cette logique là, et non dans une logique 
purement "réaliste" qu'il faudrait comprendre la phrase "Dans l'exercice de notre leadership, nous respecterons les valeurs, le jugement et les intérêts de nos amis et partenaires. Néanmoins, nous serons prêts à agir seuls quand nos intérêts et nos responsabilités uniques le demandent $»^{43}$. C'est-à-dire de s'attribuer la prérogative du souverain, celle de décider du moment où le droit doit être suspendu, et donc de façon sous-entendue, d'étendre la souveraineté des Etats-Unis à un territoire indéterminé, mondial.

C'est en ce sens que l'on peut parler avec Pierre Hassner de mentalité impériale : il s'agit pour les Etats-Unis de considérer leur souveraineté comme inviolable: «notre constitution nous a bien servi » (our constitution has served us well) ${ }^{44}$, tout en s'arrogeant le droit, au nom de conditions d'exception à l'échelle internationale ${ }^{45} \mathrm{~d}$ 'intervenir dans les affaires d'autres Etats ${ }^{46}$, voire d'opérer des "changements de régime». L'état d'exception international serait donc cette logique, contenue dans la stratégie américaine de suspendre les normes internationales (au sens du droit, des lois) tout en ayant la prétention de mener des actions qui auraient force de loi.

\section{Les trois étapes de la logique de sécurité impériale}

La logique impériale de la sécurité contenue dans le National Security Strategy s'articule donc autour de trois idées. Tout d'abord la remise en question des ordres établis de la scène internationale : que l'on croie à l'anarchie du système international et donc aux vertus de l'équilibre des puissances, ou que l'on croie au contraire à la primauté du droit international comme mode de régulation internationale, on ne peut en aucun cas penser l'action préemptive. L'action préemptive présuppose la remise en question de ces deux principes de régulation et d'interprétation du fonctionnement du système international. La stratégie américaine n'est cependant ni d'ignorer le droit international, ni de s'y soumettre. C'est une attitude différente : il s'agit de soumettre les autres Etats à un droit international remodelé sans s'y soumettre soi-même. Pour cela, cependant, il faut trouver une légitimation suffisamment forte. Cette légitimation se fait par la guerre contre le terrorisme, qui s'inscrit alors dans le registre de la guerre juste. Mais la guerre juste telle qu'elle est pensée par Grotius ne permet pas, nous l'avons vu, de légitimer la doctrine américaine : les différents chapitres du De Jure Belli ac Pacis tendent, au contraire, à démasquer le peu d'originalité de la démarche actuelle des Etats-Unis, c'est-à-dire la logique impériale.

C'est donc dans une logique impériale inavouée qu'il faut trouver - sans grande surprise sans doute - les fondements théoriques de la doctrine américaine de sécurité, et cette logique impériale peut se comprendre, dans un contexte où les séparations entre l'interne et l'externe sont abolies, comme l'extension à l'échelle internationale de l'idée d'état d'exception telle qu'elle est développée par Carl Schmitt. Une question finale pourrait donc être, avec Raymond Aron et Pierre Hassner, de se demander jusqu'à quel point la " République Impériale » américaine est capable de soutenir cette logique, sans que les appels au retour à la paganité ${ }^{47}$ de Robert Kaplan ne soient pris au sérieux et que la "barbarisation du bourgeois $»^{48}$ ne devienne définitive. Philip K. Dick, quant à lui, imagine une fin tragique à sa nouvelle puisque le préfet Anderton est obligé de s'exiler sur Mars après avoir compris la perversion d'une croyance aveugle dans l'efficacité de la brigade Précrime. 


\section{NOTES}

1. Le rapprochement entre les textes de Philip K. Dick et les discours et pratiques sécuritaires actuelles n'est pas nouveau, je suis débiteur principalement de Didier Bigo, et notamment de son article : «La recherche proactive et la gestion du risque », Déviance et société, 1997, vol 21 4, pp. 423-429, mais également des discussions que j'ai pu avoir avec Christian Olsson, Emmanuel-Pierre Guittet, Philippe Bonditti, Stéphane Davidshofer et sans doute d'autres personnes que j'oublie ici : je tiens à les remercier chaleureusement. Ce texte est imprégné de ces réflexions collectives. Merci également à Michele Manzolini et Federico Ferrone.

2. Saint Augustin, La cité de Dieu, Livre IV, Chap. IV.

3. Dick P..K., « How to build a Universe that doesn't fall apart two days later », I Hope I Shall Arrive Soon, 1986.

4. Bush G.W., The White House, National Security Strategy of the United States of America, Septembre 2002, disponible sur http://www.whitehouse.gov/nsc/nss.pdf, abrégée dorénavant sous la forme NSS.

5. Dick P.K., « Rapport Minoritaire », in Minority Report et autres récits, Paris, Gallimard, 2002, p. 18.

6. Dick P.K., ibid., p. 18, en italique dans le texte.

7. On voit bien dans cet univers de machines et de cartes perforées la croyance dans la puissance infaillible de l'informatique, jusqu'à croire en la capacité à prévoir le futur. Il est difficile de ne pas rapprocher cet univers avec les méthodes - et l'espoir - de certains courants des relations internationales (behavioristes) de prévoir les comportements des Etats.

8. "We cannot let our enemies strike first", Bush G.W., The White House, National Security Strategy of the United States of America, septembre 2002, p. 15, http://www.whitehouse.gov/ nsc/nss.pdf.

9. Hassner P. , «Etats-Unis: l'empire de la force ou la force de l'empire », Cahiers de Chaillot, $n^{\circ} 54$, septembre 2002.

10. NSS, p. 15.

11. Ibid., p. 14.

12. Ibid., p. 14.

13. Ibid., p. 15.

14. Ibid., p. 13.

15. Ibid., p. 15.

16. Hassner P., op. cit., 2002, pp. 9-10.

17. NSS, p. 31.

18. Ibid., p. 1.

19. "And, as a matter of common sense and self-defense, America will act against such emerging threats before they are fully formed ", NSS, p. iv.

20. "Some worry that it is somehow undiplomatic or impolite to speak the language of right and wrong. I disagree. Different circumstances require different methods, but not different moralities ", NSS, p. 3.

21. Edelman M., «The construction and uses of political enemies » in Constructing the Political Spectacle, Chicago, University of Chicago Press, 1998.

22. Pour une étude détaillée de la formation des courants de pensée en matière de politique étrangère américaine, voir Hassner P., Vaïsse J., Washington et le monde, dilemmes d'une superpuissance, Paris, Ed. Autrement, 2003, p. 32.

23. "Aucune disposition de la présente Charte ne porte atteinte au droit naturel de légitime défense, individuelle ou collective, dans le cas où un Membre des Nations Unies est l'objet d'une agression armée, jusqu'à ce que le Conseil de sécurité ait pris les mesures nécessaires pour 
maintenir la paix et la sécurité internationales. Les mesures prises par des Membres dans l'exercice de ce droit de légitime défense sont immédiatement portées à la connaissance du Conseil de sécurité et n'affectent en rien le pouvoir et le devoir qu'a le Conseil, en vertu de la présente Charte, d'agir à tout moment de la manière qu'il juge nécessaire pour maintenir ou rétablir la paix et la sécurité internationales ", Charte des Nations Unies, disponible sur le site internet www.un.org .

24. Murswiek D., «The American Strategy of Preemptive War and International Law », AlbertLudwigs Universität Institute of Public Law Papers und Preprints, Mars 2003, disponible sur le site : www.jura.uni-freiburg.de/ioeffr3/papers/papers.htm

25. NSS p. 15.

26. « We build a world of justice, or we will live in a world of coercion », NSS, p. 9.

27. "The reasons for our actions will be clear, the force measured, and the cause just ", NSS, p. 16.

28. Knight C., "Essential Elements Missing in the National Security Strategy of 2002 ", Cambridge, MA, Commonwealth Institute Project on Defense Alternatives Commentary, Novembre 2002. Disponible sur le site http://www.comw.org/qdr/0210knight.html.

29. "Les anciens n'avaient rien tant à cœur que de n'entreprendre aucune guerre qui ne fût juste », Grotius H., Du Droit de la Guerre et de la Paix, PUF, Paris, 1999, p. 162.

30. Ibid., p. 164.

31. Ibid., p. 166.

32. Ibid., p. 167.

33. Ibid., p. 176.

34. Hassner P., "Le Barbare et le Bourgeois, entretien avec Pierre Hassner ", Politique Internationale, été $1999, \mathrm{n}^{\circ} 84, \mathrm{pp} .81-98$.

35. NSS, p. 15.

36. Ibid., p. 163.

37. Schmitt C., cité dans Agamben G., «L'état d'exception », Le Monde, 12 Décembre 2002.

38. Walker R.B.J., Inside/Outside: International Relations as Political Theory, Cambridge, Cambridge UP, 1993.

39. "Transform America's National Security Institutions to Meet the Challenges and Opportunities of the Twenty-First Century ", NSS, p. 29.

40. Comme en témoigne l'institution du nouveau Department for Homeland Security après les attentats du 11 Septembre 2001.

41. Schmitt C., La Notion de Politique, Paris, Flammarion, 1992, p. 85.

42. Des logiques de militarisation de la police (dans les discours ou dans les dispositifs policiers) ou de d'attribution de tâches de police à l'armée (de plus en plus courants dans les domaines du peace-keeping, mais avec d'autres précédents plus funestes, comme les armées d'occupation).

43. «In exercising our leadership, we will respect the values, judgement, and interests of our friends and partners. Still, we will be prepared to act part when our interests and unique responsabilities require ", NSS, p. 31 .

44. Sous-entendu : nous ne nous plions ni aux juridictions internationales ni à tout autre instance juridique qui irait contre notre constitution.

45. Et alors tout le discours sur l'interdépendance est retourné dans une logique de contrôle impérial.

46. « Where governments find the fight against terrorism beyond their capacities, we will match their willpower and their resources with whatever help we and our allies can provide », NSS, p. 7. 47. Kaplan R.D., Warrior Politics : Why Leadership Demands a Pagan Ethos, Random, House, New York, 2001.

48. Hassner P., « Le Barbare et le Bourgeois, entretien avec Pierre Hassner », op. cit. 


\section{RÉSUMÉS}

L'oeuvre de Philip K. Dick a consisté avant tout à imaginer et à mettre en scène, à partir de données du présent, la possibilité de différents mondes sociaux. La croyance aveugle de l'Homme en une science humaine prédictive, les enjeux de pouvoir liés à la connaissance, l'idée que la « réalité » puisse être un construit - et un construit manipulable - sont autant de problèmes que l'auteur de Rapport Minoritaire partage sans le savoir avec les débats et les réflexions épistémologiques en Relations Internationales. Cet article propose une lecture de la Stratégie de Sécurité Nationale des Etats-Unis (National Security Strategy) à travers la nouvelle de science fiction de Philip K. Dick, en s'arrêtant sur l'articulation de trois idées : l'idée de l'attaque "préemptive", l'idée de la guerre juste et enfin l'idée de l'état d'exception à l'échelle internationale. Sans grande surprise sans doute, la façon dont ces idées s'articulent permettra de mettre en lumière la dimension non seulement unilatérale mais bien impériale de la logique de sécurité des Etats-Unis.

Philip K. Dick's work consisted in imagining from present data the possibility of different social worlds. The human and blind belief in a predictive human science, the power stakes linked to knowledge, the idea that « reality » can be built - and manipulated - are problems the author of Minority Report shares, without knowing it, with the International Relations debates and epistemological reflections. This article suggests a reading of the US National Security Strategy through Philip K. Dick's new Science Fiction, by articulating three ideas: the idea of a preemptive attack, the idea of a fair war and finally the idea of the state of exception at the international scale. The way these ideas articulate allow, with no surprise, to put the light on the not only unilateral but also imperial security logic of the United States.

\section{INDEX}

Mots-clés : National Security Strategy, attaque préemptive, guerre juste, sécurité

\section{AUTEUR}

\section{FRANCESCO RAGAZZI}

Francesco RAGAZZIest doctorant à l'IEP de Paris, en Sociologie des relations internationales. Il travaille sur les usages sociaux et les stratégies gouvernementales liées à la notion de « diaspora ». 